\title{
PREFACE
}

\section{Attention: Its Neuroanatomical Bases, Forms, Disorders, Assessment, and Treatment in Adults with Specific Neurological Disorders}

Of the various areas of cognition that may be compromised by acquired neurological trauma or disease, attention deficits are perhaps the most pervasive. ${ }^{1}$ Attention, like other cognitive constructs, is viewed as a multifaceted cognitive system reliant on the complex and dynamic interactions of numerous brain regions, including cortical, subcortical, and brainstem structures. Consequently, patients representing the spectrum of acquired neurogenic communication disorders commonly have attention deficits, although the qualitative and quantitative natures of these deficits can vary among the different patient populations. Because of the diversity of attention deficits that can occur in patients with brain damage, clinicians must be able to differentiate these attention disorders and design interventions accordingly. Knowledge of attention functions and disorders is also important because other aspects of cognition (e.g., working memory, inhibition, language) depend on the integrity of attention, ${ }^{2,3}$ placing patients with attention disorders at risk for a variety of other cognitive impairments.

The purpose of this issue of Seminars in Speech and Language is to provide a comprehensive overview of attention and attention disorders that will serve as an informational foundation for assessing and treating patients with neurogenic communication impairments and attention deficits. In the first article, Filley discusses the neuroanatomical representation of attention with delineation of two major neural networks proposed to support attention functioning and common neurological syndromes that may result when one or both of these networks is compromised by brain damage. Next, O'Donnell describes psychological constructs viewed as principal components of attention and how disturbances in these attention functions are manifested in patients with neurological damage and disease.

After the articles on the neuroanatomical and psychological substrates of attention, there are articles that summarize attention research with respect to specific neurogenic patient populations. In the first of these articles, Murray discusses the relationship between attention and language deficits in patients with aphasia and procedures for assessing and treating attention problems when they occur with aphasia. Cherney then reviews terminology, theory, and clinical procedures for left unilateral neglect associated with right hemisphere damage, which is one of the most common, and perhaps most complex, attention disorders. Then, Stierwalt and Murray focus on procedures for diagnosing and remediating attention problems following traumatic brain injury. In the final article, Foldi, LoBosco, and Schaefer provide a review of the growing literature on the types of attention problems associated with Alzheimer's disease and how these attention problems might contribute to the other cognitive sequelae of this progressive disease.

Attention: Its Neuroanatomical Bases, Forms, Disorders, Assessment, and Treatment in Adults with Specific Neurological Disorders; Editors in Chief, Nancy Helm-Estabrooks, Sc.D., and Nan Bernstein Ratner, Ed.D.; Guest Editor, Laura L. Murray, Ph.D. Seminars in Speech and Language, volume 23, number 2, 2002. ${ }^{1}$ Department of Speech and Hearing Sciences, Indiana University, Bloomington, Indiana. Copyright (C) 2002 by Thieme Medical Publishers, Inc., 333 Seventh Avenue, New York, NY 10001, USA. Tel: +1(212) 584-4662. 0734-0478,p;2002,23;02,087,088,ftx,en;ss100109x. 
It is anticipated that after reading this issue of Seminars, clinicians and researchers will have an improved understanding of the variety of attention functions and disorders that are intimately related to neurogenic communication disorders and outcomes. In addition, it is hoped that unresolved issues pertaining to attention and attention dysfunction raised by each of the contributing authors will lead to further delineation and advancement of attention theory and clinical practice.

Laura Murray, Ph.D. Guest Editor ${ }^{1}$

\section{REFERENCES}

1. Hochstenbach J, Mulder T, van Limbeek J, Donders $\mathrm{R}$, Schoonderwaldt $\mathrm{H}$. Cognitive decline following stroke: a comprehensive study of cognitive decline following stroke. J Clin Exp Neuropsychol 1998;20: 503-517

2. Cowan N. An embedded-processes model of working memory. In: Miyake A, Shah P, eds. Models of Working Memory: Mechanisms of Active Maintenance and Executive Control. Cambridge: Cambridge University Press; 1999:62-101

3. Crosson B. Systems that support language processes: attention. In: Nadeau S, Rothi L, Crosson B, eds. Aphasia and Language: Theory to Practice. New York: Guilford Press; 2000:372-398 